\title{
Effect of multivitamin supplements on weight gain during pregnancy among HIV-negative women in Tanzania
}

\author{
Freeman T. Changamire*, Ramadhani S. Mwiru ${ }^{\dagger}$, Karen E. Peterson ${ }^{\dagger \ddagger}$, \\ Gernard I. Msamanga ${ }^{\S}$, Donna Spiegelman ${ }^{\text {T*** }}$, Paul Petraro ${ }^{\dagger}$, Willy Urassa ${ }^{\dagger \dagger}$ and \\ Wafaie W. Fawzi ${ }^{\text {tqł }}$ \\ *Department of Family Medicine, Tufts University School of Medicine, Boston, Massachusetts, USA, `Department of Nutrition, Harvard School of Public \\ Health, Boston, Massachusetts, USA, ‡Human Nutrition Program, Department of Environmental Health Sciences, University of Michigan, Ann Arbor, \\ Michigan, USA, ${ }^{\circledR}$ Department of Community Health, Muhimbili University of Health and Allied Sciences, Dar es Salaam, Tanzania, "Department of \\ Epidemiology, Harvard School of Public Health, Boston, Massachusetts, USA, **Department of Biostatistics, Harvard School of Public Health, Boston, \\ Massachusetts, USA, t'Department Microbiology and Immunology, Muhimbili University of Health and Allied Sciences, Dar es Salaam, Tanzania, and \\ 拉Department of Global Health and Population, Harvard School of Public Health, Boston, Massachusetts, USA
}

\begin{abstract}
Multivitamin supplementation has been shown to reduce the risk of low birthweight. This effect could be mediated through gestational weight gain. However, the effect of multivitamin supplementation on weight gain during pregnancy has not been fully studied. The objective of this study was to examine the effects of multivitamins on pregnancy weight gain. We enrolled 8468 HIV-negative women from Dar es Salaam, Tanzania, in a randomised, placebo-controlled trial of multivitamins on birth outcomes. Women were randomly assigned to receive either a daily oral dose of multivitamin tablets or a placebo and were weighed every 4 weeks from enrolment until the last visit before delivery. Intent-to-treat analyses were carried out to examine the effects of multivitamins on pregnancy weight gain. Multivariate linear and binomial regression models with the log-link function were used to examine the association of weight gain during pregnancy to birthweight. The overall total weight gain was $253 \mathrm{~g}$ (SE: 69, P: 0.0003) more, while the overall 4 weekly weight gain was $59 \mathrm{~g}$ greater (SE: 18 , $P: 0.005)$ among women who received multivitamins compared to placebo. Women in the lowest quartile of gestational weight gain had babies with an average birthweight of $3030 \mathrm{~g}$ (SD: 524), while women in the highest quartile had babies weighing $3246 \mathrm{~g}$ (SD: 486), on average. Prenatal multivitamin supplements increased gestational weight gain, which was a significant predictor of birthweight.
\end{abstract}

Keywords: weight, pregnancy, multivitamins, birthweight, Africa.

Correspondence: Freeman T. Changamire, Department of Family Medicine, Tufts University School of Medicine, 136 Harrison Avenue, Boston, MA 02111, USA. E-mail: freeman.changamire@tufts.edu

\section{Introduction}

Low birthweight is a major determinant of neonatal mortality rates, which are still very high globally, especially in South Asia and sub-Saharan Africa (Ashworth 1998; WHO 2006). Inadequate weight gain during pregnancy is a major risk factor for low birthweight (Kramer 1987; Stein et al. 1995; WHO 1997; Neufeld et al. 1999). Improving maternal nutritional status before or during pregnancy could help prevent low birthweight and neonatal deaths in developing countries.

Evidence on the relationship between macronutrients and prenatal weight gain is evolving. Supplementation trials during pregnancy in developing countries have shown that total energy intake increases the rate of prenatal weight gain (Qureshi et al. 1973; Lechtig et al.1975; Tontisirin et al. 1986; Prentice et al. 1987) and that isocaloric protein supplementation decreases maternal weight gain (Kramer 2000). 
Overall, however, the impact of macronutrient food supplementation on gestational weight gain appears to depend on the prior energy deficit, the impact being greater for those with low pre-pregnancy weight for height or in those women where food intake has been restricted (Institute of Medicine 1990, 2009). The effect of individual micronutrients such as zinc, iron and folate on pregnancy outcomes including birthweight has been looked at before (Goldenberg et al. 1995; Rasmussen 2001; Fall et al. 2003). However, there are a few studies that have examined the effect of multiple micronutrients supplementation on pregnancy weight gain. In one study among HIV-positive women in Tanzania, we showed that multivitamin supplementation increases pregnancy weight gain (Villamor et al. 2002). Another study conducted in Chile showed that women given a daily fortified milk product had higher weight gain in pregnancy than women who were given unfortified powdered milk (Mardones-Santander et al. 1988). Among women in Mexico, multivitamin supplements did not increase weight gain during pregnancy after adjusting for baseline differences in body mass index (BMI; Ramakrishnan et al. 2005).

We previously reported that multivitamins increased birthweight in a randomised trial of HIVnegative women (Fawzi et al. 2007). In that study, the incidence of low birthweight was $7.8 \%$ among infants in the multivitamin group and $9.4 \%$ among those in the placebo group [relative risk (RR), 0.82; $95 \%$ confidence interval (CI): 0.70, 0.95]. The mean birthweight difference between the two groups was $67 \mathrm{~g}$ $(P<0.001)$. In this paper, we examined the effect of multivitamin supplements in the same trial on pregnancy weight gain, and assessed the relationship of gestational weight gain to birthweight.

\section{Subjects and methods}

\section{Study design and population}

We analysed data from 8468 HIV-negative women who participated in a randomised, double-blind, placebo-controlled trial in Dar es Salaam, Tanzania. Women were randomised between August 2001 and July 2004. The objectives of the trial were to determine if daily multivitamin supplementation during the prenatal period would reduce the incidence of fetal loss, low birthweight $(<2500 \mathrm{~g})$ and preterm birth ( $<37$ weeks). The details of the study population for this trial have been described elsewhere (Fawzi et al. 2007). Consonant with standard prenatal care in Tanzania, women in both treatment groups also received daily supplements of $0.25 \mathrm{mg}$ of folic acid, $60 \mathrm{mg}$ of ferrous sulphate and malaria prophylaxis in the form of sulfadoxine-pyrimethamine tablets.

The participants were screened and recruited from women who attended antenatal clinics in Dar es Salaam. The basic eligibility requirements were a negative test for HIV infection, gestational age between 12 and 27 weeks according to the date of the last menstrual period, 18 years of age or older and an intention to deliver and stay in Dar es Salaam for at least 1 year after delivery. HIV type 1 serologic status was ascertained from all the women who consented to participate in the trial by means of two sequential enzyme-linked immunosorbent assay tests (Urassa et al. 1999). All women provided written informed consent to participate. Women were randomly assigned to receive either a daily oral dose of multivitamin tablets containing $20 \mathrm{mg}$ of vitamin $\mathrm{B}_{1}, 20 \mathrm{mg}$ of vitamin $\mathrm{B}_{2}, 25 \mathrm{mg}$ of vitamin $\mathrm{B}_{6}, 100 \mathrm{mg}$ of niacin, $50 \mu \mathrm{g}$ of vitamin $\mathrm{B}_{12}, 500 \mathrm{mg}$ of vitamin $\mathrm{C}, 30 \mathrm{mg}$ of

\footnotetext{
Key messages

- The effect of multivitamin supplementation on weight gain during pregnancy has not been fully studied

- Multivitamin supplementation during pregnancy appeared to improve gestational weight gain and gestational weight gain is a predictor of birthweight.

- The effect of multivitamins on birthweight might be mediated through increase in gestational weight gain.

- Further research is required to find the optimal weight gain in mothers in developing countries, and the effects of intervening earlier in pregnancy on gestational weight and on the risks of adverse pregnancy outcomes.
} 
vitamin $\mathrm{E}$ and $0.8 \mathrm{mg}$ folic acid or a placebo from enrolment until delivery.

Trained research nurses administered a questionnaire to all the women at enrolment during the second trimester to collect baseline data on the woman's age, partner's age, maternal education, marital status, parity, socioeconomic status and other behavioural and reproductive factors. In addition, nurses obtained a complete medical and obstetric history at baseline and performed clinical examinations at baseline and monthly.

Height was measured at baseline using a stadiometer to the nearest $0.1 \mathrm{~cm}$ with the subjects without head covers and shoes. Weight was measured to the nearest $100 \mathrm{~g}$ using balance scales with the women wearing light clothing without shoes.

Women were encouraged to deliver at Muhimbili Hospital, where research midwives attended to the delivery and weighed the infants to the nearest $10 \mathrm{~g}$ on a standard beam balance immediately after birth.

\section{Data analyses}

Analyses were limited to women who had a singleton pregnancy, a known date of pregnancy outcome and at least two weight measurements from enrolment through the end of gestation. Data from women with fetal deaths were not excluded from the analyses. Compared with 779 women whose data were not included in this analysis, women who were included were significantly older, taller, had greater mid upper arm circumference (MUAC), lower gestational age at entry and lower haemoglobin. The distribution of the baseline characteristics was compared across the treatment groups by using the Wilcoxon rank sum and the Kruskal-Wallis test for continuous variables and $\chi^{2}$ tests for proportions. Intent-to-treat analyses were carried out to examine treatment effects on weight gain.

\section{Effect of supplements on gestational weight gain}

Changes in weight gain during pregnancy were examined in two ways: (1) as the total weight change over the period of follow-up [presented as means and standard deviations (SDs)]; (2) as 4-week changes in weight from one visit to the next controlling for the actual length of the inter-visit interval.

The weight difference between two consecutive visits $\left(\triangle W E I G H T_{i j}\right)$ was modelled as a continuous response variable with the treatment assignment as the explanatory variable across the repeated measures in each individual using a generalised estimating equation approach (Diggle et al. 2002), according to the following model, $\triangle W E I G H T_{i j}=\beta_{0}+\beta_{2} \mathrm{EXP}_{i}+$ $b_{i}+e_{i j}$

Where $\mathrm{E}\left(b_{i}\right)=\mathrm{E}\left(e_{i j}\right)=0$, Var $\left(b_{i}\right)=\sigma_{\mathrm{B}}^{2}$ and Var $\left(e_{i j}\right)=\sigma_{\mathrm{W}}^{2}, W E I G H T_{i j}$ is the weight measurements during follow-up $(j)$ for subject $(i), E X P_{i}$ is the treatment assignment for subject $(i), b_{i}$ is the betweenperson random error, $e_{i j}$ is the within-individual random error, $\sigma_{\mathrm{B}}^{2}$ is the between-person variance and $\sigma_{\mathrm{W}}{ }^{2}$ is the within-person variance. Robust estimators of the variance were used to construct CI and $P$-values in the analysis, and the working correlation structure allowed for negative correlations between successive pairs of measurements as would be expected for a dependent variable of this form.

Models for the effect of multivitamin supplementation on total weight gain controlled for gestational age at randomisation and time from the first weight measurement to the last weight measurement. Trimester specific analyses were done using data in each trimester. Only women having at least two weight measurements in each trimester were included. For the weight difference analysis, this means that intervals beginning in the second trimester and ending in the third trimester were excluded. The analysis of weight gain as a mediator between multivitamin supplementation and birthweight used the method of Lin, Fleming and DeGruttola (Lin et al. 1997). We also assessed whether the effect of supplements was modified by baseline characteristics, including BMI at entry to this study, haemoglobin $\left(<11, \geq 11 \mathrm{~g} \mathrm{dL}^{-1}\right)$, cluster of differentiation 4 (CD4) count as a marker of immune status with the median as the cut-off $\left(<775, \geq 775\right.$ cells $\left.\mathrm{mm}^{-3}\right)$ and malaria infection (malaria at baseline vs. no malaria), prematurity (defined as birth before 37 weeks of gestation.), the calendar months of the first and last measurements and the number of weight measurements. Modification of the effect of multivitamin supplementation on 
the total weight change was examined by adding the interaction terms of multivitamin supplementation with the potential modifier to a model with the main effects and testing by the robust score test.

\section{Birth outcomes in relation to rate of weight gain}

The association between total weight gain and birthweight was analysed using regression models controlling for gestational age at first weight measurement, time from first to last weight measurement, and time from last weight measurement to delivery, as well as the multivitamin group. The possibly non-linear relationship between weight gain and birthweight was examined non-parametrically with restricted cubic splines (Durrleman \& Simon 1989) in a model adjusted as described above.

To examine the relationship between gestational weight gain and birthweight outcome, linear regression was used with birthweight as a continuous variable and total weight gain categorised in quartiles. We adjusted for multivitamins regimen, BMI at randomisation, height, marital status, parity, number of weight measurements, gestational age at delivery and fetal sex.

We assessed whether the association between pregnancy weight gain and the risk of low birthweight was modified by baseline BMI at entry to this study. The statistical significance of this interaction was tested using the likelihood ratio test. Tests for non-linearity used the robust score test for the continuous birthweight outcome and the likelihood ratio test for the dichotomised outcome, comparing the model with only the linear term to the model with both the linear and the cubic spline terms. Analyses were carried out with Statistical Analyses System software, Version 9 (SAS Institute Inc., Cary, NC, USA).

Continuous variables are presented as mean (SD). Estimates based on models are presented as estimate (95\% CI). Categorical variables are summarised as per cents. Effects on binary outcomes are summarised as RRs with $95 \% \mathrm{CI}$. All $P$-values are two-sided. A $P$-value $\leq 0.05$ is considered significant. No adjustments were made for multiple comparisons.

\section{Results}

A total of 8468 HIV-negative women were randomised, but only 7689 women with singleton pregnancies and at least two weight measurements during pregnancy were included in the analysis. Of these, 7378 women had live births, and the 7354 with known birthweights were included in the birthweight analyses. The characteristics of our study population are shown in Table 1 . The distribution of baseline covariates, including the socio-demographic characteristics, did not differ significantly across treatment regimens. The mean gestational age at randomisation was 21 weeks (SD: 3.5 ) and the last visit before delivery occurred at 36 weeks (SD: 3.9 ) on average. The average time from last weight measurement to delivery was 3 weeks (SD: 2.7), and this did not differ

Table I. Maternal characteristics at baseline according to treatment assignment*

\begin{tabular}{|c|c|c|}
\hline \multirow[t]{3}{*}{ Characteristic } & Placebo & Multivitamin \\
\hline & Mean (SD) & Mean (SD) \\
\hline & $(n=3935)$ & $(n=3933)$ \\
\hline Age (years) & $25.1(5.1)$ & $25.2(5.1)$ \\
\hline Height (cm) & $155.3(7.5)$ & $155.4(8.0)$ \\
\hline Weight (kg) & $59.4(10.6)$ & $59.6(10.5)$ \\
\hline $\mathrm{BMI}\left(\mathrm{kg} \mathrm{m}^{-2}\right)$ & $24.6(4.0)$ & $24.6(3.8)$ \\
\hline MUAC (cm) & $26.5(4.3)$ & $26.6(4.0)$ \\
\hline Education (years) & $7.1(2.8)$ & $7.1(2.9)$ \\
\hline Parity & $1.0(1.3)$ & $1.0(1.3)$ \\
\hline Wealth score ${ }^{\dagger}$ & $1.6(1.0)$ & $1.6(1.0)$ \\
\hline $\begin{array}{l}\text { Gestational age at recruitment } \\
\text { (weeks) }\end{array}$ & $21.2(3.5)$ & $21.3(3.5)$ \\
\hline $\begin{array}{l}\text { Gestational age at last weight } \\
\text { measurement (weeks) }\end{array}$ & $36.3(3.9)$ & $36.4(3.9)$ \\
\hline CD4 count (cells $\mathrm{mm}^{-3}$ ) & $804(262)$ & $807(251)$ \\
\hline Haemoglobin (g/100 mL) & $10.3(1.6)$ & $10.2(1.5)$ \\
\hline Total energy intake (kcal) & $2130(866)$ & $2129(865)$ \\
\hline Protein intake $(\mathrm{g})$ & $55(34)$ & $55(33)$ \\
\hline Carbohydrate intake (g) & $351(139)$ & $351(138)$ \\
\hline Fat intake $(\mathrm{g})$ & $68(42)$ & $68(43)$ \\
\hline $\begin{array}{l}\text { Time from randomisation to first } \\
\text { diet measurement (weeks) }\end{array}$ & $7.6(1.7)$ & $7.6(1.7)$ \\
\hline $\begin{array}{l}\text { Time from last weight } \\
\text { measurement to delivery } \\
\text { (weeks) }\end{array}$ & $3.0(2.7)$ & $3.1(2.9)$ \\
\hline
\end{tabular}

CD4, cluster of differentiation 4; MUAC, mid upper arm circumference; SD, standard deviation. *None of the baseline characteristics were significantly different between the treatment groups. ${ }^{\dagger}$ FilmerPritchett wealth score. 
Table 2. Estimated total weight gain and weight gain by trimester for women in placebo and multivitamin arms*

\begin{tabular}{|c|c|c|c|c|c|c|}
\hline \multirow[t]{2}{*}{ Outcome } & \multirow[t]{2}{*}{$N$} & Placebo & \multirow[t]{2}{*}{$N$} & Multivitamins & \multirow[t]{2}{*}{ Difference* (SE) } & \multirow[t]{2}{*}{$P$-value } \\
\hline & & Mean (SD) & & Mean (SD) & & \\
\hline \multicolumn{7}{|l|}{ Estimated total weight gain (g) } \\
\hline $\begin{array}{l}\text { Overall: from baseline to } \\
\text { last visit }{ }^{\dagger}\end{array}$ & 3749 & $5248(5150,5346)$ & 3835 & $5501(5397,5605)$ & $253(177,388)$ & 0.0003 \\
\hline Second trimester & 2758 & $1316(1214,1418)$ & 2753 & $1400(1304,1496)$ & $84(-23,191)$ & 0.12 \\
\hline Third trimester & 3407 & $2618(2549,2686)$ & 3408 & $2743(2671,2814)$ & $125(30,220)$ & 0.001 \\
\hline \multicolumn{7}{|c|}{ Estimated 4 week weight gain $(\mathrm{g})^{\ddagger}$} \\
\hline Overall & 3749 & $1319(1291,1348)$ & 3735 & $1379(1349,1408)$ & $59(18,100)$ & 0.005 \\
\hline Second trimester & 2758 & $1395(1347,1443)$ & 2753 & $1447(1399,1496)$ & $52(-19,123)$ & 0.15 \\
\hline Third trimester & 3407 & $1316(1280,1351)$ & 3408 & $1385(1348,1422)$ & $70(18,121)$ & 0.008 \\
\hline
\end{tabular}

$\mathrm{SD}$, standard deviation. *Based on generalized estimating equations (GEE) models controlling for gestational age at first weight and the time from to last weight measurement (estimated at the median values of the covariates for each time group). ${ }^{\dagger}$ From 21 to 36 weeks of gestation, on average. ${ }^{\ddagger}$ Based on GEE models controlling for time between measurements.

across the treatment regimens. There were 4.6 weight measurements per woman, independent of treatment assignment and $95 \%$ of the women had three or more measurements. The women with $\geq 2$ weight measurements were comparable between the multivitamins and the placebo groups with respect to baseline characteristics.

The effects of multivitamins on weight gain are shown in Table 2. Multivitamin supplementation increased the overall total weight gain by $253 \mathrm{~g} \mathrm{(95 \%}$ CI: 177-388). The total weight gain from randomisation to the last visit during pregnancy was $5501 \mathrm{~g} \mathrm{(95 \%}$ CI, 5397-5605) in the multivitamin group, compared to $5248 \mathrm{~g}$ (95\% CI: 5150-5346) in the placebo group. The overall 4-weekly weight gain in the multivitamins group, 1379 g (95\% CI: 1349-1408), was significantly higher than that in the placebo group, 1319 (95\% CI: 1291-1348). There was no difference in total weight gain or 4-weekly weight gains between placebo and multivitamin groups in the second trimester; however, there was a significant difference between the placebo and the supplementation groups in both the total weight gain, $125 \mathrm{~g}(P=0.001)$ and the 4-weekly weight gains $70 \mathrm{~g}(P=0.0008)$ in the third trimester. We found no significant interactions between multivitamins and baseline BMI $(P>0.10)$ Table 3 .

There were 7378 women with live births. Of these, 7354 had infants with known birthweights. The relationship between total gestational weight gain and birthweight was linear as shown in Fig. $1(P=0.001)$.
Table 3. Effects of multivitamin supplementation on gestational weight gain stratified by body mass index (BMI) at baseline*

\begin{tabular}{lrlll}
\hline $\begin{array}{l}\text { BMI category } \\
\left(\mathrm{kg} \mathrm{m}^{-2}\right)\end{array}$ & $N$ & $\begin{array}{l}\text { Effect } \\
\text { estimate }^{\dagger}(\mathrm{kg})\end{array}$ & $P$-value & $\begin{array}{l}P \text { for } \\
\text { interaction }\end{array}$ \\
\hline$<22$ & 1890 & 0.17 & 0.17 & 0.41 \\
$22-<25$ & 2391 & 0.22 & 0.07 & \\
$25-29$ & 2005 & 0.39 & 0.005 & \\
$\geq 30$ & 656 & 0.23 & 0.39 & \\
\hline
\end{tabular}

*Baseline was 21 weeks of gestational age, on average. Weight gained by those in the multivitamin group - weight gained by those in the placebo group based on generalized estimating equations (GEE) models.

In the multivariate analysis, women in the lowest quartile of gestational weight gain had babies with an average birthweight of $3030 \mathrm{~g}$ (SD: 524), while women in the highest quartile gain had babies weighing an average of $3246 \mathrm{~g}$ (SD: 486). Baseline BMI did not modify the relationship between gestational weight gain and birthweight. Weight gain mediates 6.4\% (95\% CI: $2.2,10.7 \%$ ) of the effect of multivitamin supplementation on birthweight.

\section{Discussion}

We described the pattern of weight gain during pregnancy among HIV-negative women in Tanzania and examined the relationship between the rates of weight gain in pregnancy with birthweight. Multivitamin supplementation resulted in increases in both 


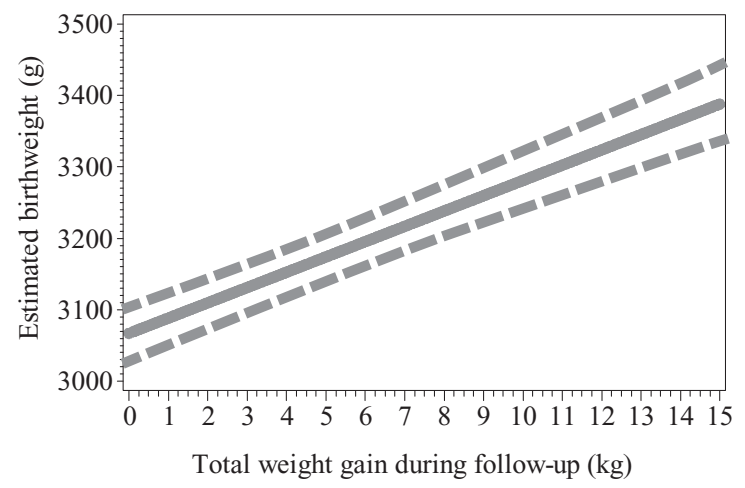

Fig. I. Birthweight in relation to total gestational weight gain.

overall total weight gain (253 g; $95 \%$ CI: 177-388) and the 4-weekly weight gain (59 g; 95\% CI: $18-100)$ in pregnancy. There was a significant difference between the placebo and the supplementation groups in both the total weight gain and the 4-weekly weight gain in the third trimester but not in the second trimester. Taken together, these findings may suggest that the effect of multivitamin supplements on gestational weight gain may be more pronounced during the third trimester than the second trimester. If confirmed in other studies, this may be reassuring as many women in Tanzania and other developing countries do not present to antenatal care, and hence be afforded the benefit of micronutrient supplementation, until later in the second trimester.

Multivitamins could improve the rate of weight gain in pregnancy through various mechanisms including improving the immune status that reduces the risk of infections (Bendich 2001). Furthermore, by restoring normal metabolism, multivitamins may restore the feeling of well-being, which may result in improved appetite (Committee on Nutrition 1958; Major et al. 2008). Furthermore, multivitamins particularly thiamine and $\mathrm{B}$ vitamins have a role in protein and energy metabolism, which could affect fetal growth by improving the fetus' handling of macronutrients (Ashworth \& Antipatis 2001), and result in higher maternal weight gain during pregnancy. Another mechanism, through which multivitamins could improve fetal growth, is via the antioxidant nature of vitamins such as $\mathrm{C}$ and $\mathrm{E}$. There are studies that have shown that maternal oxidative stress plays an important role in the pathophysiology of low birthweight (Scholl \& Stein 2001). Micronutrients might also improve birth outcomes by improving placental development and function (Ashworth \& Antipatis 2001).

Our findings are in accord with other studies that have examined the effect of multivitamin supplementation on the rate of weight gain during pregnancy. A study that was conducted in a similar population in Tanzania in HIV-positive women showed that multivitamin supplementation resulted in an increase in maternal weight gain during the third trimester (304 g; 95\% CI: 17, 590) and a significant 30\% reduction in the risk of weight loss. A study in Greece suggested that increases in serum vitamin $\mathrm{C}$ could lead to a higher total weight gain (Kafatos et al.1989). In a supplementation trial in Chile which randomised women received a milk-based product fortified with thiamine, pyridoxine, niacin, vitamins $\mathrm{A}, \mathrm{C}$ and $\mathrm{E}$ or a non-fortified milk product, total weight gain was significantly greater in the intervention group (Mardones-Santander et al. 1988). Among women in Mexico, however, multivitamin supplements did not increase weight gain during pregnancy after adjusting for baseline differences in BMI (Ramakrishnan et al. 2005).

We showed that an increase in rate of weight gain during pregnancy increases birthweight; however, the effect of the supplements on gestational weight gain explained only about $6 \%$ of the effect on birthweight.

There are studies that have shown that low rate of weight gain in pregnancy is associated with low birthweight (Abrams \& Selvin 1995; Hickey et al. 1996; Berenson et al. 1997). Some studies, however, have shown that large gains in pregnancy weight are not always beneficial in terms of improving birthweight outcomes (Hickey et al. 1997; Nielsen et al. 2006). Most of these studies documented excessive weight gain to have adverse effects such as macrosomia with a higher risk for birth injury (Johnson et al. 1992), increased risk of operative delivery (Young \& Woodmansee 2002) and increased risk for maternal morbidity particularly postpartum weight retention (Parker \& Abrams 1993). In our study, the relationship between gestational weight gain and birthweight within the range of total weight gain of most of the 
women in our study (0-15 kg) was linear. The association of gestational weight gain and birthweight was not modified by baseline BMI.

A strength of our study is that its large size gave ample statistical power to examine the research questions of interest. One limitation, however, was that women were largely enrolled in the middle of the second trimester, which limited our ability to examine at the effect of multivitamins in pregnancy from conception through the first trimester and the ability to study weight changes in the first trimester and early second trimester.

In conclusion, multivitamin supplementation during pregnancy increased gestational weight gain among HIV-negative women in Tanzania. Birthweight increased with increased gestational weight gain. Further research is required to find the optimal weight gain in mothers in developing countries, and the effects of intervening earlier in pregnancy on gestational weight and on the risks of adverse pregnancy outcomes.

\section{Acknowledgements}

We thank the women who participated in this project; the study coordinator, Illuminata Balonzi; the study physician, Heavington Mshiu; and the research assistants, laboratory technicians, nurses, midwives and administrative staff who made the study possible. We also acknowledge the valuable input from Ellen Hertzmark.

\section{Source of funding}

This work has been supported by the National Institutes of Child Health and Human Development (NICHD R01 37701). FTC was supported by the Irene M. and Fredrick J. Stare Nutrition Education Fund.

\section{Conflicts of interest}

The authors declare that they have no conflicts of interest.

\section{Contributions}

FTC conducted the data analyses, interpreted the data and wrote the initial draft of the manuscript.
RSM assisted in manuscript revisions, GIM, WU and PP participated in the study implementation and field supervision. KEP contributed to the study design, interpreted the data and revised the initial draft. DS provided statistical guidance in the study design and data analyses and WWF was the principal investigator, contributed to the study design and implementation, and interpreted the data. All authors contributed to the editing of the final version of the manuscript.

\section{References}

Abrams B. \& Selvin S. (1995) Maternal weight gain pattern and birth weight. Obstetrics and Gynecology 86, 163-169.

Ashworth A. (1998) Effects of intrauterine growth retardation on mortality and morbidity in infants and young children. European Journal of Clinical Nutrition $\mathbf{5 2}$ (Suppl. 1), S34-S41; discussion S41-S42.

Ashworth C.J. \& Antipatis C. (2001) Micronutrient programming of development throughout gestation. Reproduction 122, 527-535.

Bendich A. (2001) Micronutrients in women's health and immune function. Nutrition 17, 858-867.

Berenson A.B., Wiemann C.M., Rowe T.F. \& Rickert V.I. (1997) Inadequate weight gain among pregnant adolescents: risk factors and relationship to infant birth weight. American Journal of Obstetrics and Gynecology 176, 1220-1224; discussion 1224-1227.

Committee on Nutrition (1958) APPRAISAL of the use of vitamins B1 and B12 as supplements promoted for the stimulation of growth and appetite in children. Pediatrics. 21, 860-864.

Diggle P.J., Heagerty P., Liang K.-Y. \& Zeger S.L. (2002) Analysis of Longitudinal Data. Oxford University Press: Oxford, UK.

Durrleman S. \& Simon R. (1989) Flexible regression models with cubic splines. Statistics in Medicine 8, 551561.

Fall C.H., Yajnik C.S., Rao S., Davies A.A., Brown N. \& Farrant H.J. (2003) Micronutrients and fetal growth. The Journal of Nutrition 133,1747S-1756S.

Fawzi W.W., Msamanga G.I., Urassa W., Hertzmark E., Petraro P., Willett W.C. et al. (2007) Vitamins and perinatal outcomes among HIV-negative women in Tanzania. The New England Journal of Medicine 356, 14231431.

Goldenberg R.L., Tamura T., Neggers Y., Copper R.L., Johnston K.E., DuBard M.B. et al. (1995) The effect of zinc supplementation on pregnancy outcome. The Journal of American Medical Association 274, 463-468. 
Hickey C.A., Cliver S.P., McNeal S.F., Hoffman H.J. \& Goldenberg R.L. (1996) Prenatal weight gain patterns and birth weight among nonobese black and white women. Obstetrics and Gynecology 88, 490-496.

Hickey C.A., McNeal S.F., Menefee L. \& Ivey S. (1997) Prenatal weight gain within upper and lower recommended ranges: effect on birth weight of black and white infants. Obstetrics and Gynecology 90, 489-494.

Institute of Medicine (1990) Nutrition During Pregnancy report. pp. 6-7.

Institute of Medicine (2009) Nutrition During Pregnancy report. p. $137 \&$ pp. $146-148$.

Johnson J.W., Longmate J.A. \& Frentzen B. (1992) Excessive maternal weight and pregnancy outcome. American Journal of Obstetrics and Gynecology 167, 353-370; discussion 370-372.

Kafatos A.G., Vlachonikolis I.G. \& Codrington C.A. (1989) Nutrition during pregnancy: the effects of an educational intervention program in Greece. The American Journal of Clinical Nutrition 50, 970-979.

Kramer M.S. (1987) Determinants of low birth weight: methodological assessment and meta-analysis. Bulletin of the World Health Organization 65, 663-737.

Kramer M.S. (2000) Isocaloric balanced protein supplementation in pregnancy. Cochrane Database of Systematic Reviews (Online) (2), CD000118.

Lechtig A., Delgado H., Lasky R., Yarbrough C., Klein R.E., Habicht J.P. et al. (1975) Maternal nutrition and fetal growth in developing countries. American Journal of Diseases of Children (1911) 129, 553-556.

Lin D.Y., Fleming T.R. \& De Gruttola V. (1997) Estimating the proportion of treatment effect explained by a surrogate marker. Statistics in Medicine 16, 1515-1527.

Major G.C., Doucet E., Jacqmain M.S., Onge M., Bouchard C. \& Tremblay A. (2008) Multivitamin and dietary supplements, body weight and appetite: results from a crosssectional and a randomised double-blind placebocontrolled study. The British Journal of Nutrition 99 $1157-1167$.

Mardones-Santander F., Rosso P., Stekel A., Ahumada E., Llaguno S., Pizarro F. et al. (1988) Effect of a milk-based food supplement on maternal nutritional status and fetal growth in underweight Chilean women. The American Journal of Clinical Nutrition 47, 413-419.

Neufeld L., Pelletier D.L. \& Haas J.D. (1999) The timing of maternal weight gain during pregnancy and fetal growth. American Journal of Human Biology 11, 627637.

Nielsen J.N., O'Brien K.O., Witter F.R., Chang S.C., Mancini J., Nathanson M.S. et al. (2006) High gestational weight gain does not improve birth weight in a cohort of African American adolescents. The American Journal of Clinical Nutrition 84, 183-189.
Parker J.D. \& Abrams B. (1993) Differences in postpartum weight retention between black and white mothers. Obstetrics and Gynecology 81, 768-774.

Prentice A.M., Cole T.J., Foord F.A., Lamb W.H. \& Whitehead R.G. (1987) Increased birth weight after prenatal dietary supplementation of rural African women. The American Journal of Clinical Nutrition 46, 912-925.

Qureshi S., Rao N.P., Madhavi V., Mathur Y.C. \& Reddi Y.R. (1973) Effect of maternal nutrition supplementation on the birth weight of the newborn. Indian Pediatrics 10, 541-544.

Ramakrishnan U., González-Cossío T., Neufeld L.M., Rivera J. \& Martorell R. (2005) Effect of prenatal multiple micronutrient supplements on maternal weight and skinfold changes: a randomized double-blind clinical trial in Mexico. Food and Nutrition Bulletin 26, 273-280.

Rasmussen K. (2001) Is there a causal relationship between iron deficiency or iron-deficiency anemia and weight at birth, length of gestation and perinatal mortality? The Journal of Nutrition 131, 590S-601S; discussion 601S-603S

Scholl T.O. \& Stein T.P. (2001) Oxidant damage to DNA and pregnancy outcome. The Journal of Maternal-Fetal Medicine 10, 182-185.

Stein A.D., Ravelli A.C. \& Lumey L.H. (1995) Famine, third-trimester pregnancy weight gain, and intrauterine growth: the Dutch Famine Birth Cohort Study. Human Biology 67, 135-150.

Tontisirin K., Booranasubkajorn U., Hongsumarn A. \& Thewtong D. (1986) Formulation and evaluation of supplementary foods for Thai pregnant women. The American Journal of Clinical Nutrition 43, 931-939.

Urassa W., Godoy K., Killewo J., Kwesigabo G., Mbakileki A., Mhalu F. et al. (1999) The accuracy of an alternative confirmatory strategy for detection of antibodies to HIV-1: experience from a regional laboratory in Kagera, Tanzania. Journal of Clinical Virology 14, 25-29.

Villamor E., Msamanga G., Spiegelman D., Antelman G., Peterson K.E., Hunter D.J. et al. (2002) Effect of multivitamin and vitamin A supplements on weight gain during pregnancy among HIV-1-infected women. The American Journal of Clinical Nutrition 76, 1082-1090.

WHO (1997) A WHO collaborative study of maternal anthropometry and pregnancy outcomes. International Journal of Gynaecology and Obstetrics 57, 1-15.

WHO (2006) Neonatal and Perinatal Mortality: Country, Regional and Global Estimates. World Health Organization: Geneva, Switzerland.

Young T.K. \& Woodmansee B. (2002) Factors that are associated with cesarean delivery in a large private practice: the importance of prepregnancy body mass index and weight gain. American Journal of Obstetrics and Gynecology 187, 312-318; discussion 318-320. 